\title{
3. UPSTREAM RESEARCH TO SUPPORT THE GPELF (BASIC RESEARCH)
}

\subsection{PATHOGENESIS}

\author{
Christopher L. King and James W. Kazura**
}

\section{Summary of Prioritized Research Needs}

1) Develop revised, standardized set of definitions for lymphatic and urogenital pathology seen with LF,

2) Use animal models and clinical studies to define influence of LF parasites, molecules, endosymbionts, and hostinduced factors on lymphatic endothelium,

3) Define role of co-infections (local bacterial or systemic TB, HIV/AIDS, etc.) in pathogenesis of LF disease,

4) Define effects of the LF-altered host responsiveness on reactions to vaccines or to other infectious agents (malaria, HIV/AIDS, TB, etc.),

5) Explore roles of innate and adaptive immune responses to LF in the initiation, progression or reversal of lymphatic disease and disease of other organs,

6) Define the role of the host immune response in the mechanism of action of the common antifilarial drugs (especially DEC),

7) Define differential host susceptibility to development of disease caused by genetic, endocrinologic, nutritional, and other types of host heterogeneity.

\subsubsection{Overview}

Why study pathogenesis?

It is, of course, knowledge that leads to solutions for problems, and nowhere is there a better example than in the management of lymphatic filarial disease. Once thought beyond repair and clinically hopeless, the pathology of LF is now approached with extremely effective tools and strategies that were all developed because of remarkable advances in understanding the pathogenesis of filarial disease (see Section 2.3). Further understanding will undoubtedly improve clinical management still more, and better definition of the underlying molecular mechanisms of disease should also lead to wholly novel strategies for both diagnosis and treatment.

Current global efforts to eliminate LF focus principally on the reduction of transmission with the progressive lowering of endemicity levels. How such changes impact on the development or progression of disease makes understanding the mechanisms of its pathogenesis extremely important. On the one hand, there have been reports of the regression of LF disease (lymphedema and hydrocoele/lymphocoele) following MDA aimed only at interrupting transmission. ${ }^{1}$ On the other hand, potential scenarios exist for ways in which changes in LF transmission might undesirably affect the evolution of disease. For example, 1) reduction in levels of endemicity following MDA might lead to diminished immunoregulation and consequent exaggeration of disease progression after re-infection; 2) repeated treatment itself might

** Other contributors in this working group are listed in Annex 2. trigger exaggerated host immune responses to filarial products that could enhance progression of disease and result in persistent lymphatic damage; and 3) even with a decrease in overall prevalence of infection persistent, low-level infections could result in altered immunoregulatory and/or inflammatory responses that could, in turn, affect an individual's susceptibility to other types of infection, reduce responsiveness to vaccines or even impair a child's growth and development. Finally, it is also important to understand the mechanisms underlying pathogenesis since the consequences of LFinitiated damage to the lymphatics may persist long after infection has been eliminated. It is unknown to what degree this lymphatic damage is reversible, and it is clear that such damaged lymphatics predispose individuals, by some undefined mechanism, to prolonged periods of increasingly damaging lymphedema and recurrent bacterial infections.

\section{Barriers to studying lymphatic pathology.}

Numerous obstacles have persistently hindered studies on LF pathogenesis. First, it has often been difficult even to establish whether a particular clinical syndrome is or is not associated with LF, principally because of the difficulty in definitively establishing current or prior infection. Confusion arising from the fact that other conditions may produce similar pathology or that multiple pathogenic pathways may lead to the same clinical endpoint often can be dispelled by longitudinal studies of patient populations, ${ }^{2}$ but such studies are particularly difficult for LF because of the long period of time between infection and development of clinically overt disease. Second, the uncertainties regarding LF pathogenesis have been compounded by the difficulty in obtaining appropriate human tissues and by inadequate tools for detecting subclinical lymphatic abnormalities. Third, there has not been a good animal model of disease for LF, particularly for $W$. bancrofti.

Recent developments or improvements in imaging technologies have now given much better access to patient pathology, such that, at least at the organismic level models for understanding the pathogenesis of LF are being developed. $^{3-5}$ The current advances in proteomic and genomic technologies now promise to help overcome some of the remaining barriers and lead to additional refinement and progress in developing a unified model for understanding LF pathogenesis at the molecular, as well as the organismic, level.

\subsubsection{Research Needs}

Defining the issues.

In addition to the broad issues of pathogenesis already identified (i.e., need for improved markers of morbidity/ pathology, relation between endemicity and LF disease, effect 
of MDAs or specific treatment on disease progression, role of co-infection in pathogenesis) and the micro-issues related to actual pathogenic mechanisms (described later in this report), there is one particularly important concern that transcends these issues; namely, the need for a revised and standardized set of definitions for LF disease.

For example, acute filarial lymphangitis, while thought to be the reaction to a dying or damaged adult worm, lacks a standard approach to distinguish it from an episode caused by bacterial infection in an individual chronically affected by LF. ${ }^{6}$ Similarly better definitions for urogenital disease are needed. Hydrocoele may be mistaken for scrotal lymphedema or unreduced hernia by an inexperienced examiner, but ultrasonography can greatly improve the precision and accuracy of its diagnosis. ${ }^{7}$ Many studies do not distinguish a simple hydrocoele from chylocele, but this difference has extremely important clinical implications. Other examples of terms that require better clinical distinctions are subclinical versus clinical disease; lymphatic disease versus lymphatic dysfunction. Better characterizations of patient populations are also needed. Therefore,

- a consensus conference should be held to establish agreed definitions for the different manifestations of LF disease and for the diagnostic criteria distinguishing them.

\section{Identifying external factors affecting pathogenesis.}

Adult worms.

The direct impact of adult worms and their endosymbiont Wolbachia spp. on the host is poorly understood, particularly regarding the lymphatic endothelium. A striking feature of infected individuals is the marked lymphatic dilation observed surrounding the aggregations of adult worms. This dilation can extend well beyond the location of the worms themselves, suggesting the presence of a diffusible factor produced by the worms. ${ }^{6,8}$ An important research challenge, then, is

- to identify factors released by adult worms that cause lymphatic dilation, especially using new technologies (such as proteomics).

The recent identification of lymphatic-specific vascular endothelial growth factors (VEGF-C and VEGF-D) and molecular cell surface markers such as the VEGFR-3 receptor and podoplanin have made it possible to isolate and culture lymphatic endothelial cells (LEC) without loss of differentiated properties. ${ }^{9}$ This could provide a powerful in vitro model to study the effects of excretory/secretory products of adult worms on LEC. The VEGFR-3 triggers an important signaling pathway for LEC proliferation and survival. This and related receptors would be valuable tools for studying the protein-protein interactions with excretory-secretory molecules that were identified by proteomic analysis, then screened by computational methods and tested directly. ${ }^{10}$ In vivo models using severe combined immunodeficient (SCID) mice and gerbils with LF that develop lymphangeictasia could complement in vitro studies with the added power of being able to examine the effect of specific signaling pathways through targeted gene deletion. The degree to which lymphangiectasia is reversible following removal of the worms/growth factors could also be studied in these models. Therefore,
- in vitro models based on cultured lymphatic endothelial cells should be established and exploited to define the mechanisms of lymphangiectasis,

- in vivo models using LF-infected SCID mice and gerbils should be studied in parallel with the in vitro models to define mechanisms of LEC stimulation and the signaling pathways involved in causing lymphangiectasia.

Little is known about the microanatomy of lymphatic endothelium and the degree to which host inflammation contributes to lymphatic pathology, especially following death of adult worms. ${ }^{8}$ Such studies have been severely limited by the difficulty in obtaining pathology specimens in humans. However,

- animal models and earlier-collected pathology samples from patients should now be reassessed using the newer molecular tools available.

Differences in parasite strains may contribute to differences in host pathology, but very little is known is this area. Therefore,

- identification of microsatellite or other DNA markers and statistical techniques necessary to make inferences regarding the parasite population within the host and in the human population should be undertaken, so that correlation between different parasite strains and disease pathogenesis can be investigated.

\section{Bacterial microflora.}

Bacterial infections aggravate pre-existing pathology in animal models. Lymphatic dysfunction in humans predisposes to secondary infection which can be recognized clinically to accelerate lymphatic damage, ${ }^{6,11}$ but the degree to which bacterial microflora contributes to disease and whether this varies among populations remains poorly understood. Therefore,

- studies need to be carried out in humans and in animal models to address the important question of the role of bacterial superinfection in the pathogenesis of LF disease.

Non-infectious toxic elements.

In parts of the world with certain soil compositions, walking barefoot results in absorption of silica that can cause lymphatic damage and non-filarial elephantiasis. ${ }^{12}$ Such soils, with silica or potentially other substances toxic to the lymphatics, might be important cofactors for the development of additional lymphatic pathology in some LF-endemic populations. Therefore,

- the mechanisms underlying this soil-induced lymphatic damage should be defined, along with its role in aggravating LF-induced lymphatic disease.

\section{Co-infections and LF disease.}

Many individuals with LF are also infected with malaria, HIV, tuberculosis, and/or other helminths. Such concurrent infections might affect the host immune response by impairing immunoregulation to LF and thereby promoting disease through chronic infection. Conversely, LF may also down- 
modulate host responses to these same infections and attenuate the disease they cause. Epidemiologic studies in southeast Asia have reported that concurrent helminth infections may reduce the frequency of severe of malaria morbidity. ${ }^{13,14}$ While such studies would suggest the possibility that control of LF and other chronic helminth infections might actually increase the frequency of severe malaria morbidity, other reports from Africa describe just the opposite; namely, that the elimination of helminth infection significantly decreases the frequency of malaria attacks. ${ }^{15,16}$ Therefore,

- the impact of LF control on the clinical expression of other co-existing infections needs thorough investigation.

\section{Host nutrition.}

Lymphatic filariasis typically occurs among the poorest individuals in underdeveloped regions where malnutrition is rampant. Malnutrition can affect host immune responses as well as growth and repair mechanisms in tissues. Serum protein levels also decrease with malnutrition. This situation could affect intravascular and tissue oncotic pressures that would accentuate tissue fluid accumulation from lymphatic dysfunction and, additionally, provide further substrate for bacterial infection. Obesity, too, is a growing problem in some developing countries owing to a lack of available nutritious foods. The excess weight can affect the hemodynamic lymphatic flow especially in the lower extremities and contribute to a greater risk for chronic lymphedema in LF-infected individuals. Thus,

- the role of host nutrition should be investigated as a possible co-factor in the pathogenesis of lymphatic disease related to LF.

\section{Identifying host-defense factors affecting pathogenesis.}

Several stages of the parasite persist chronically within the host and continuously release antigens. The type and magnitude of the chronic immune stimulation by these antigens contribute substantially to the spectrum of disease observed in LF. The respective roles of innate and adaptive host immune responses-and their relative contributions in initiation, progression and reversal of lymphatic disease, despite being one of the most intense areas of research on pathogenesis of LF, still remains poorly understood.

\section{Innate immune response.}

The recent identification of Wolbachia as an essential endosymbiont of filarial parasites present in all stages has sparked greater interest in a potential role for innate immune responses in disease development. ${ }^{17,18}$ Wolbachia are gramnegative bacteria that produce molecules capable of directly stimulating cells of the innate immune system and, if released in sufficient quantities, may produce symptoms that might mimic gram-negative bacterial infections. The degree to which living adult worms or mf express or release Wolbachia molecules remains unknown. More likely, Wolbachia molecules are released upon death of the parasites. Indeed, Wolbachia molecules may play a prominent role in acute filarial lymphangitis, presumably triggered by spontaneous death of adult worms or following treatment. Although mf appear to contain less Wolbachia antigen, there is likely to be some continuous destruction of $\mathrm{mf}$ and the consequent release of Wolbachia antigens. The effect of these Wolbachia antigens on the innate immune response may also influence the adaptive immune response to filarial antigens. Research studies are needed, therefore,

- to identify parasite or Wolbachia molecules responsible for inducing host innate immune responses,

- to determine the effect of these activated innate immune responses on the adaptive immune response of the host,

- to assess the role that innate or adaptive responses to Wolbachia have in the pathogenesis of acute filarial fevers or other inflammatory syndromes.

\section{Adaptive immune responses-immune regulation.}

Central to the immunopathogenesis of LF is the development of immune regulation. Successful immunoregulation limits the collateral damage to the host. The mechanisms that initiate and sustain this immune regulation remain incompletely understood, but they are clearly important to consider because mass chemotherapy programs alter the dynamics of transmission and the burdens of infection in affected communities.

Hyporesponsiveness. Most studies indicate that the immune hyporesponsiveness that develops to filariae is antigenspecific, suggesting that there are either diminished frequencies of filarial antigen-specific $\mathrm{T}$ cells or an increase of immunoregulatory $\mathrm{T}$ cells (or both). ${ }^{19,20}$ The idea of immune tolerance, either by clonal deletion or anergy of antigen-specific $T$ cells from prenatal or early antigen exposure was first proposed more than 30 years ago, ${ }^{21}$ but remains to be directly tested. Mass treatment that reduces infection levels in women of child-bearing age might significantly reduce this form of immune regulation. The recent identification of natural and antigen-specific $\mathrm{T}$ regulatory cells in humans raises the interesting possibility that these cells might be expanded in LF and contribute prominently to immunoregulation.

Some studies, particularly those in areas of high transmission, indicate a component of non-specific immunosuppression. ${ }^{22}$ The possibility that parasite molecules actively suppress the host immune response has been shown in vitro, ${ }^{23-25}$ but whether this occurs in vivo remains poorly understood. Some parasite-derived molecules have been identified to have homology to host immunoregulatory cytokines in different stages of the parasite. Overall, how these parasite-derived molecules affect host cell function remains unknown but is likely to be relevant to other chronic helminth infections as well. With the sequencing of the filarial genome nearing completion and annotation actively underway, the opportunity to identify additional molecules using bioinformatics or proteomics approaches should greatly enhance this line of research. The respective roles of molecules derived from different parasite stages, e.g., L3, mf, or adult worms, will be of particular interest.

The possibility that the parasite itself, particularly adult stages of the parasite, co-opts host molecules on its tegument that either disguise it from the host immune response (i.e., molecular mimicry) or impair host effector mechanisms (e.g., acquisition of decay accelerating factors that block the complement cascade) has received little study in filariasis. These and similar mechanisms help protect adult schisto- 
somes within the human host and permit it to survive for decades. ${ }^{26}$ It is conceivable that adult filarial worms may have evolved similar survival strategies.

Necessary research to address these immunoregulatory issues will require investigation

- to define the specific mechanisms underlying the immune down-regulation induced by LF infection and to gauge their effects on other concurrent infections,

- to identify the changes in immune regulation induced by MDA activities and assess their impact on the pathogenesis of filarial or other diseases,

- to define the pre-natal influence on filarial-specific immune regulatory mechanisms and the effect that MDA activities have on them,

- to characterize the parasite-derived molecules responsible for down-regulation or evasion of the host's immune response.

Hyperresponsiveness. More recognizably related to disease is the host immune response to $\mathrm{mf}$ that account for the vast burden of parasite material in the host. Exaggerated host response to $\mathrm{mf}$ is thought to be the basis of the tropical pulmonary eosinophilia (TPE) syndrome, an allergy-like clinical manifestation of LF where $\mathrm{mf}$ are rapidly cleared in the lung. ${ }^{27,28}$ It is possible that even partial immunity to $\mathrm{mf}$ may accelerate clearance of this stage of the parasite, but that this accelerated clearance, although beneficial in reducing transmission, is achieved at the cost of an increased host pathogenic response to dying mf. Valuable research studies would include

- identification of the mechanisms involved in triggering allergic responsiveness to the parasite,

- efforts to understand the mechanisms underlying the control of allergic responsiveness to LF parasites in essentially all situations except for TPE.

Host genetics and susceptibility to disease.

Differences in response patterns among individuals could also be driven by genetic diversity in the host immune response, ${ }^{29}$ including not only that related to the human leukocyte antigen gene complex, but also to common variants in other genes that regulate the host immune response.

Genetic diversity in the regulation of lymphatic endothelial function may also predispose an individual to lymphatic disease. Several hereditary conditions have already been linked with specific genetic defects. Milroy's disease, which is one form of hereditary lymphedema, has been associated with a missense mutation in the gene encoding VEGFR-3, demonstrating that an intact VEGFR-3 signal is required for normal lymphatic endovascular function. ${ }^{9}$ Another form of hereditary lymphedema referred to as lymphedema-distichiasis syndrome is caused by mutations in the transcription factor FOXC2. ${ }^{30}$ Studies should be carried out

- to examine the hypothesis that polymorphisms in these genes or their promoters would be associated with greater risk of lymphedema with LF.

Previous studies have indicated a genetic contribution to LF disease based on human epidemiologic investigations, ani- mal models, and association studies. The recent sequencing of the human genome has provided further opportunity to explore in greater detail the genetic susceptibility to human disease. Particularly useful would be genome-wide linkage studies (family studies, disease markers) or genetic association studies using a case-control design that examines candidate single nucleotide polymorphisms or haplotypes. Such genetic information might help to identify populations at increased risk for disease that might benefit from more aggressive intervention strategies, as well as providing leads for the further development of anti-parasitic drugs and vaccines. Therefore,

- such epidemiologic studies should be undertaken to define a potential genetic determinant of LF susceptibility or disease.

\section{Endocrinology.}

Gender and age differences in rates of infection and disease suggest possible hormonal effects on LF. Most striking are the localization and concentration of adult worms in the lymphatic vessels in the spermatic cord. This concentration of parasites is likely an important factor contributing to the risk for developing hydrocoele or lymphocoele in men. Though poorly understood, adult worms also concentrate in the inguinal lymphatics of both sexes. It is possible that worms might be attracted by specific tissue derived hormones.

A number of studies in many different endemic areas indicate that men are more susceptible to infection and disease than women. ${ }^{31}$ An increased male susceptibility to infection has also been observed in the Mongolian jird with B. malayi. ${ }^{32}$ This differential susceptibility may be regulated by both immunologic and non-immunologic mechanisms; however, the reasons for these differences remain obscure. One possibility is that sex-specific hormones may influence susceptibility to infection, parasite survival, and/or disease. Therefore,

- a more detailed understanding of the role of hormones in susceptibility for infection and disease should be explored for developing novel disease interventions.

\section{Molecular tools to assess pathogenesis.}

A research opportunity with particular potential derives from the recent advances in expression and functional proteomics to identify biomarkers for disease. Specific molecules have been shown to be elevated in certain pathologic conditions, and these can be identified in serum using such technologies as protein microarrays or mass spectroscopy. Indeed these assays have been useful in studying several types of cancer and could potentially be developed for LF. A clinically relevant application of protein arrays that might apply particularly to LF is the identification of proteins that induce antibody responses in several disease states. Microarrays, produced by attaching hundreds of proteins and peptides to the surface of derivatized glass slides, could be incubated with patient serum, and fluorescent labels used to detect antibody binding to specific proteins (e.g., stage-specific proteins) that then might be associated with certain forms of disease. ${ }^{10}$ The technologies for proteomic analysis are rapidly expanding with increasing sensitivity, reduced sample requirement, lower cost, higher through-put and more effectiveness in 
identifying protein alterations (e.g., post-translation modifications). Therefore,

- studies with new molecular technologies would be extremely promising for assessing and monitoring the predisposition, occurrence or progression of LF disease syndromes.

\subsubsection{References}

1. Bockarie MJ, Tisch DJ, Kastens W, Alexander ND, Dimber Z, Bockarie F, Ibam E, Alpers MP, Kazura JW, 2002. Mass treatment to eliminate filariasis in Papua New Guinea. $N$ Engl $J$ Med 347: 1841-1848.

2. Ravindran B, 2003. Aping Jane Goodall: insights into human lymphatic filariasis. Trends Parasitol 19: 105-109.

3. Amaral F, Dreyer G, Figueredo-Silva J, Noroes J, Cavalcanti A, Samico SC, Santos A, Coutinho A, 1994. Live adult worms detected by ultrasonography in human Bancroftian filariasis. Am J Trop Med Hyg 50: 753-757.

4. Freedman DO, de Almeida Filho PJ, Besh S, Maia e Silva MC, Braga C, Maciel A, 1994. Lymphoscintigraphic analysis of lymphatic abnormalities in symptomatic and asymptomatic human filariasis. J Infect Dis 170: 927-933.

5. Witte CL, Witte MH, 1999. Diagnostic and interventional imaging of lymphatic disorders. Int Angiol 18: 25-30.

6. Dreyer G, Noroes J, Figueredo-Silva J, Piessens WF, 2000. Pathogenesis of lymphatic disease in Bancroftian filariasis: a clinical perspective. Parasitol Today 16: 544-548.

7. Tobian AA, Tarongka N, Baisor M, Bockarie M, Kazura JW, King CL, 2003. Sensitivity and specificity of ultrasound detection and risk factors for filarial-associated hydrocoeles. $\mathrm{Am} \mathrm{J}$ Trop Med Hyg 68: 638-642.

8. Figueredo-Silva J, Noroes J, Cedenho A, Dreyer G, 2002. The histopathology of bancroftian filariasis revisited: the role of the adult worm in the lymphatic-vessel disease. Ann Trop Med Parasitol 96: 531-541.

9. Karkkainen MJ, Makinen T, Alitalo K, 2002. Lymphatic endothelium: a new frontier of metastasis research. Nat Cell Biol 4: E2-E5.

10. Hanash S, 2003. Disease proteomics. Nature 422: 226-232.

11. Esterre P, Plichart C, Huin-Blondey MO, Nguyen L, 2000. Role of streptococcal infection in the acute pathology of lymphatic filariasis. Parasite 7: 91-94.

12. Onapa AW, Simonsen PE, Pedersen EM, 2001. Non-filarial elephantiasis in the Mt. Elgon area (Kapchorwa District) of Uganda. Acta Trop 78: 171-176.

13. Nacher M, Singhasivanon P, Traore B, Vannaphan S, Gay F, Chindanond D, Franetich JF, Mazier D, Looareesuwan S, 2002. Helminth infections are associated with protection from cerebral malaria and increased nitrogen derivatives concentrations in Thailand. Am J Trop Med Hyg 66: 304-309.

14. Nacher M, 2004. Interactions between worm infections and malaria. Clin Rev Allergy Immunol 26: 85-92.

15. Le Hesran JY, Akiana J, Ndiaye el HM, Dia M, Senghor P, Konnate L, 2004. Severe malaria attack is associated with high prevalence of Ascaris lumbricoides infection among children in rural Senegal. Trans R Soc Trop Med Hyg 98: 397-399.
16. Spiegel A, Tall A, Raphenon G, Trape JF, Druilhe P, 2003. Increased frequency of malaria attacks in subjects co-infected by intestinal worms and Plasmodium falciparum malaria. Trans $R$ Soc Trop Med Hyg 97: 198-199.

17. Saint Andre A, Blackwell NM, Hall LR, Hoerauf A, Brattig NW, Volkmann L, Taylor MJ, Ford L, Hise AG, Lass JH, Diaconu E, Pearlman E, 2002 The role of endosymbiotic Wolbachia bacteria in the pathogenesis of river blindness. Science 295: 1892-1895.

18. Taylor MJ, 2002. A new insight into the pathogenesis of filarial disease. Curr Mol Med 2: 299-302.

19. King CL, Kumaraswami V, Poindexter RW, Kumari S, Jayaraman K, Alling DW, Ottesen EA, Nutman TB, 1992. Immunologic tolerance in lymphatic filariasis. Diminished parasitespecific $\mathrm{T}$ and $\mathrm{B}$ lymphocyte precursor frequency in the microfilaremic state. J Clin Invest 89: 1403-1410.

20. Nutman TB, Kumaraswami V, 2001. Regulation of the immune response in lymphatic filariasis: perspectives on acute and chronic infection with Wuchereria bancrofti in South India. Parasite Immunol 23: 389-399.

21. Beaver PC, 1970. Filariasis without microfilaremia. Am J Trop Med Hyg 19: 181-189.

22. King CL, Connelly M, Alpers MP, Bockarie M, Kazura JW, 2001. Transmission intensity determines lymphocyte responsiveness and cytokine bias in human lymphatic filariasis. J Immunol 166: 7427-7436.

23. Maizels RM, Gomez-Escobar N, Gregory WF, Murray J, Zang X, 2001. Immune evasion genes from filarial nematodes. Int $J$ Parasitol 31: 889-898.

24. Semnani RT, Sabzevari H, Iyer R, Nutman TB, 2001. Filarial antigens impair the function of human dendritic cells during differentiation. Infect Immun 69: 5813-5822.

25. Semnani RT, Law M, Kubofcik J, Nutman TB, 2004. Filariainduced immune evasion: suppression by the infective stage of Brugia malayi at the earliest host-parasite interface. J Immunol 172: 6229-6238.

26. Ramalho-Pinto FJ, Carvalho EM, Horta MF, 1992. Mechanisms of evasion of Schistosoma mansoni schistosomula to the lethal activity of complement. Mem Inst Oswaldo Cruz 87 (Suppl 4): 111-116.

27. Ottesen EA, Neva FA, Paranjape RS, Tripathy SP, Thiruvengadam KV, Beaven MA, 1979. Specific allergic sensitsation to filarial antigens in tropical eosinophilia syndrome. Lancet 1: $1158-1161$.

28. Lobos E, Zahn R, Weiss N, Nutman TB, 1996. A major allergen of lymphatic filarial nematodes is a parasite homolog of the gamma-glutamyl transpeptidase. Mol Med 2: 712-724.

29. Choi EH, Nutman TB, Chanock SJ, 2003. Genetic variation in immune function and susceptibility to human filariasis. Expert Rev Mol Diagn 3: 367-374.

30. Finegold DN, Kimak MA, Lawrence EC, Levinson KL, Cherniske EM, Pober BR, Dunlap JW, Ferrell RE, 2001. Truncating mutations in FOXC2 cause multiple lymphedema syndromes. Hum Mol Genet 10: 1185-1189.

31. Brabin L, 1990. Sex differentials in susceptibility to lymphatic filariasis and implications for maternal child immunity. Epidemiol Infect 105: 335-353.

32. Ash LR, 1971. Preferential susceptibility of male jirds (Meriones unguiculatus) to infection with Brugia pahangi.J Parasitol 57: 777-780. 